\title{
ljfis
}

\section{Estimation System of Blood Pressure Variation with Photoplethysmography Signals Using Multiple Regression Analysis and Neural Network}

\author{
Seung-II Cho ${ }^{1,2}$, Takumi Negishi ${ }^{2}$, Minami Tsuchiya ${ }^{2}$, Muneki Yasuda ${ }^{2}$ and \\ Michio Yokoyama ${ }^{2}$ \\ ${ }^{1}$ Innovation Center for Organic Electronics, Yamagata University, Yamagata, Japan \\ ${ }^{2}$ Graduate School of Science and Engineering, Yamagata University, Yamagata, Japan
}

Received: Dec. 1, 2018

Revised : Dec. 13, 2018

Accepted: Dec. 21, 2018

Correspondence to: Michio Yokoyama

(yoko@yz.yamagata-u.ac.jp)

(CThe Korean Institute of Intelligent Systems

c) This is an Open Access article distributed under the terms of the Creative Commons Attribution Non-Commercial License (http://creativecommons.org/licenses/ by-nc/3.0// which permits unrestricted noncommercial use, distribution, and reproduction in any medium, provided the original work is properly cited.

\begin{abstract}
In this study, a target is to improve the accuracy of a blood pressure (BP) estimation system using photoplethysmography (PPG) signals. A BP estimation algorithm using multiple regression analysis is proposed and a BP estimation using the neural network is studied. Experimental results have shown that estimation accuracy can be improved. Estimation error of systolic BP value using multiple regression analysis with the proposed algorithm was reduced by approximately $16.3 \%$. Furthermore, estimation error was reduced by approximately $21.6 \%$ than conventional multiple regression analysis in case of a BP estimation by machine learning using the neural network. It has been found that estimation accuracy is improved and shows the possibility of BP estimation using the neural network.
\end{abstract}

Keywords: Blood pressure estimation, Multiple regression analysis, Neural network, Correlation coefficient, Photoplethysmography

\section{Introduction}

Recently, the number of patients of lifestyle-related diseases in which lifestyle habits such as eating habits, exercise habits, rest, smoking, and drinking are related to onset and progress is increasing [1, 2]. Several of these diseases, including cancer, dementia and cardiovascular disease, are now the leading causes of morbidity and mortality on individuals and societies [3. 4]. The prevention of lifestyle-related diseases is very important to save healthy living because their preliminary groups have been increasing. Lifestyle-related diseases are characterized by sudden onset without subjective symptoms until symptoms worsen. Therefore, a system that can easily manage physical condition using wearable vital sensors anytime anywhere is required for prevention of lifestyle-related diseases [5- -10$]$.

The blood pressure (BP) values are available as an indicator of daily health management. A high BP is the main cause of cardiovascular disease such as stroke and heart disease [11, 12]. This hypertension accounts for nearly half of the mortality of lifestyle-related patients. Therefore, it is effective for the prevention of lifestyle-related diseases that knowing their own BP values from daily life.

Currently BP monitors are mainly used for measurement using a cuff. However, BP 


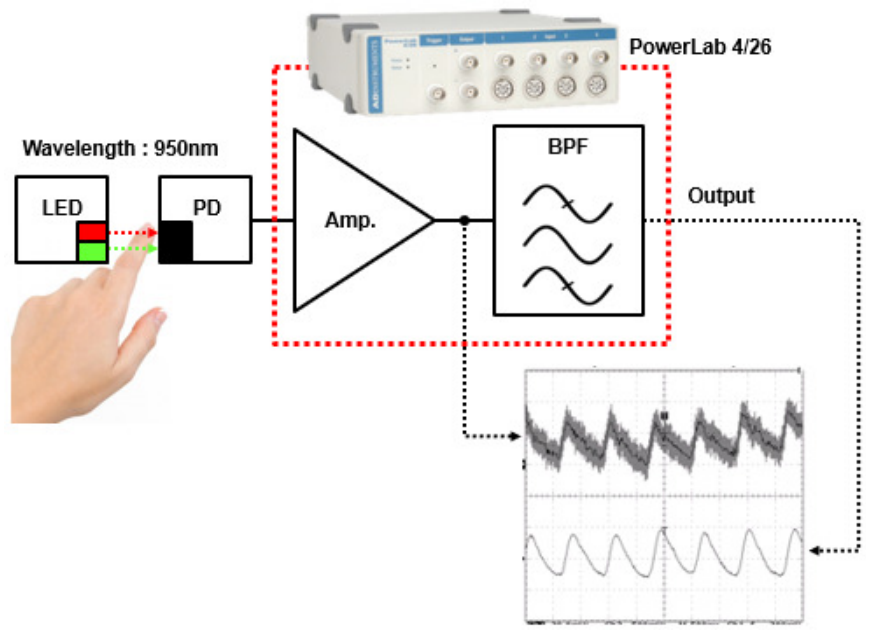

Figure 1. Conceptual diagram of the pulse wave measurement using photoplethysmography signal.

measurement using a cuff tends to neglect measurement in daily life because of unpleasant feeling of oppression at the time of measurement, troublesome cuff attachment and not suitable for carrying. Recently, there have been a few studies on handy BP measurement without a cuff such as pulse wave velocity method [13-15]. However, there is no method which is selection of parameters and designation of sections having a high correlation with the $\mathrm{BP}$ value considering the characteristics of users. Moreover, a method for highly accurate cuffless BP estimation has not been established. It is necessary to derive an estimation formula considering the characteristics of the user and to improve the estimation accuracy.

In this study, estimation system of BP variation using the photoplethysmography (PPG) signals is established. An improved algorithm of BP estimation method by multiple regression analysis is proposed and verified. Furthermore, BP estimation using the neural network is investigated.

\section{Photoplethysmography and Pulse Wave Pa- rameters}

\subsection{The Principle of Photoplethysmography}

The PPG is adopted for the BP estimation method in this study. Infrared LED/photodiode (PD) with a wavelength of $950 \mathrm{~nm}$ is utilized. The PPG is a method to observe the pulsations of the blood vessel by measuring the change in the photoabsorption of hemoglobin at the blood vessel flow. Pulsation is influenced by the transmitted or reflected light because hemoglobin with/without oxygen has remarkable absorption change mainly
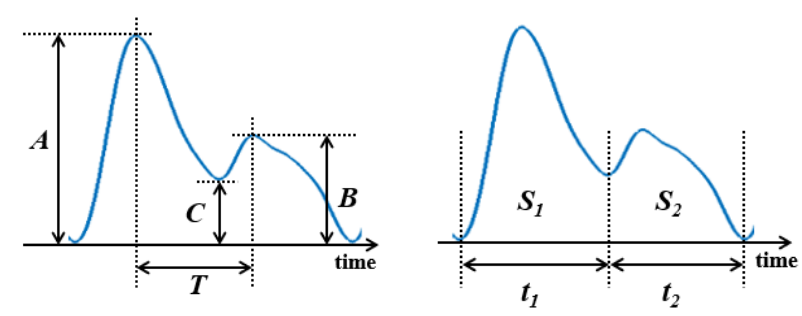

Figure 2. Pulse wave parameters.

in the near-infrared band. The PD receives the transmitted or reflected light that is not absorbed by hemoglobin. Figure 1 shows the diagram of pulse wave measurement using the PPG. Hemoglobin is irradiated by the LED light passing through a finger. The PD detects the reflection or transmission light, which includes scattering information of blood vessel corresponding to pulsation, from an infrared LED through a finger. The detected signal is then amplified and filtered at the subsequent signal processing circuits for waveform shaping [15]. In this study, the PowerLab 4/26 is used for amplifiers and filters.

\subsection{Pulse Wave Parameters}

Characteristic parameters obtained from the pulse wave are effective for estimating the BP because pulse wave is closely related to BP. Multiple regression analysis [16] with the characteristic parameters is utilized to estimate the variations of BP. The number of parameters for multiple regression analysis is selected among 16 types of pulse wave characteristic parameters, as shown in Figure 2 and Table 1.

\section{Estimation Algorithms of Blood Pressure Vari- ation}

\subsection{Estimation Algorithm Using Multiple Regression Anal- ysis}

The correlation coefficients between the BP value and each pulse wave parameter are expected to vary from person to person. However, the proposed algorithm in [15] did not consider characteristics of the users as shown in Figure 3. In this study, improved algorithm is proposed as shown in Figure 3. Parameters suitable for BP estimation are determined for each user. Furthermore, the section of the pulse wave suitable for estimation is automatically extracted in measuring the pulse wave just before the BP estimation. The multiple regression analysis is performed using parameters and section of the pulse wave. 
Table 1. Descriptions of pulse wave parameters

\begin{tabular}{|c|c|}
\hline Parameter & Description \\
\hline Pulse rate & $\begin{array}{l}\text { The number of times of rippling pulse } \\
\text { wave per minute }\end{array}$ \\
\hline$A$ & $\begin{array}{l}\text { Top of ejected wave: } \\
\text { Maximum amplitude of wave caused } \\
\text { by pumping blood from the heart }\end{array}$ \\
\hline$B$ & $\begin{array}{l}\text { Top of reflected wave: } \\
\text { Maximum amplitude of wave caused } \\
\text { by reflection at the peripheral vascular }\end{array}$ \\
\hline$C$ & $\begin{array}{l}\text { Incisura: Height of chasm which exists } \\
\text { between the ejected and reflected waves }\end{array}$ \\
\hline$R I$ & $\begin{array}{c}\text { Reflection Index: } \\
\text { Ratio of ejected and reflected waves; } \\
R I(\%)=\left(\frac{B}{A}\right) \times 100\end{array}$ \\
\hline$T$ & $\begin{array}{c}\text { Interval between tops of ejected } \\
\text { and reflected wave }\end{array}$ \\
\hline$t_{1}$ & $\begin{array}{l}\text { Interval from generation point of pulse } \\
\text { wave to incisura }\end{array}$ \\
\hline$t_{2}$ & $\begin{array}{l}\text { Interval from incisura to } \\
\text { end of pulse wave }\end{array}$ \\
\hline$t$ & $\begin{array}{l}\text { Period of one pulse wave; } \\
\qquad t=t_{1}+t_{2}\end{array}$ \\
\hline$S_{1}$ & $\begin{array}{l}\text { Partial area of one pulse } \\
\text { wave until incisura }\end{array}$ \\
\hline$S_{2}$ & $\begin{array}{l}\text { Partial area of one pulse } \\
\text { wave after incisura }\end{array}$ \\
\hline$S$ & $\begin{array}{l}\text { Area of one pulse wave; } \\
\qquad S=S_{1}+S_{2}\end{array}$ \\
\hline$E_{r}$ & $\begin{array}{l}\text { Ratio of the maximum } \\
\text { amplitude of ejected } \\
\text { wave to the incisura; } E_{r}=\frac{A}{C}\end{array}$ \\
\hline$R_{r}$ & $\begin{array}{c}\text { Ratio of the maximum } \\
\text { amplitude of reflected } \\
\text { wave to the incisura; } R_{r}=\frac{B}{C}\end{array}$ \\
\hline$t_{r}$ & Ratio of $t 1$ to $t 2 ; t_{r}=\frac{t_{1}}{t_{2}}$ \\
\hline$S_{r}$ & Ratio of $S_{1}$ to $S_{2} ; S_{r}=\frac{S_{1}}{S_{2}}$ \\
\hline
\end{tabular}

In order to select parameters and to designate sections having a high correlation with the BP value considering the characteristics of users, measured BP value and 16 types of pulse wave parameters of section $\mathrm{A}$ to $\mathrm{D}$ are tested for correlation respectively as shown in Figure 4. If the risk factor $\alpha=0.05$, parameters

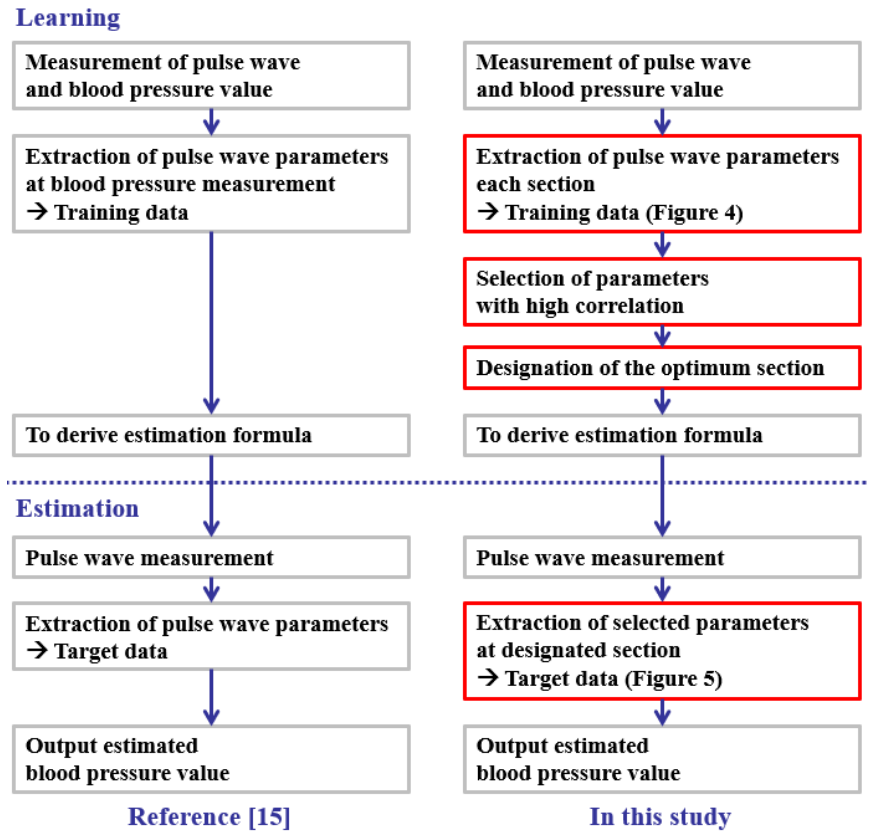

Figure 3. Comparison of algorithm.

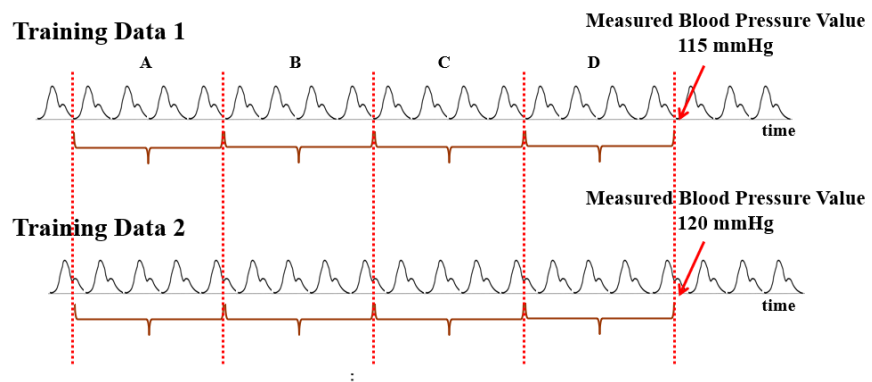

Figure 4. Selection of parameters.

tested as having correlation are extracted. Multicollinearity is examined for a combination of extracted parameters. The coefficient of determination can be derived by each estimation formula which is created by combining the parameters. If all the coefficients of determination are less than 0.5 , it is judged that the parameters are highly independent and the risk factor of multicollinearity is low.

The multiple regression analysis of selected parameters and $\mathrm{BP}$ values that is section $\mathrm{A}$ to $\mathrm{D}$ as training data is performed to derive estimation formulas. Comparing the coefficient of determination of derived estimation formula at each section, an estimation formula with the largest coefficient of determination is adopted. A section in which an estimation formula with the largest coefficient of determination can be derived is designated as the optimum section. The BP value is estimated using the 




Figure 5. Designation of the optimum section.

estimation formula in the designated optimum section of the estimation target data. For example, when the B is designated as the optimum section, the BP value is estimated using the data of section B of the estimation target data as shown in Figure 5.

\subsection{Estimation Algorithm Using Neural Network}

Figure 6 shows the NN estimation model for BP estimation using the neural network where the $x_{i}(i=1,2,3, \ldots, 16)$ is parameter values of estimation target data and the $y$ is estimated $\mathrm{BP}$ value [17]. The NN estimation model is setting of the 3 layered neural network. The input layer consists of 16 nodes. The hidden layer consists of 8 nodes. And the output layer consists of just one node. The activation functions of the hidden nodes are the sigmoid function ;

$$
a(z)=\frac{1}{1+e^{-z}}
$$

and that of the output node is the linear function;

$$
a(z)=z
$$

The training is done by the back-propagation algorithm which minimizes the mean-square-error function ;

$$
E=\frac{1}{n} \sum_{\mu=1}^{n}\left(t_{\mu}-y\left(x_{\mu}\right)\right)^{2},
$$

where training data set is $D=\left(t_{\mu}, x_{\mu}\right) \mid \mu=1,2, \ldots, n, x_{\mu}$ is input data vector $x=\left(x_{1}, x_{2}, x_{3}, \ldots, x_{16}\right)$ in $\mu$-th training data vector, $t_{\mu}$ is target data in $\mu$-th training data, $y\left(x_{\mu}\right)$ is output for input $x_{\mu}$. In addition, the NN estimation does not perform a selection of parameters, which are algorithms proposed by multiple regression analysis. Learning with the $\mathrm{NN}$ estimation model uses all the measured parameter data as training data. However, in the case of NN Esti_A, the NN estimation does not perform a designation of the optimum section. In the case of

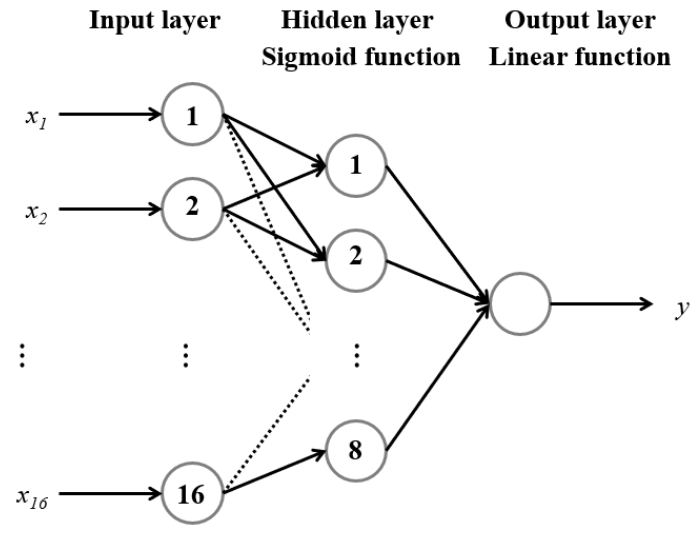

Figure 6. NN estimation model for blood pressure estimation.

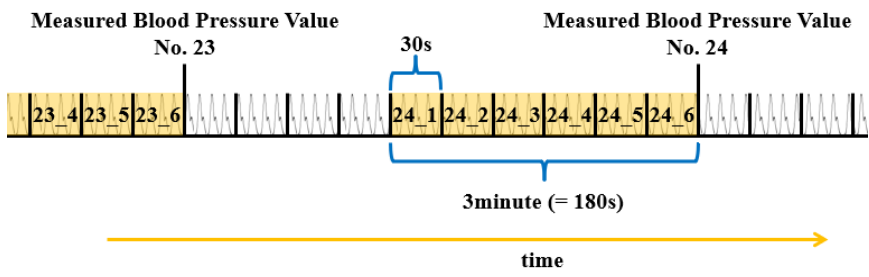

Figure 7. Divided section of pulse wave.

NN Esti_B, the NN estimation performs that. Using the learned $\mathrm{NN}$ estimation model of two cases, the BP value is estimated from the estimation target data.

\section{Experiments}

\subsection{Experimental Methodology}

The subject was a healthy adult male. The PPG signals are measured from the finger tip of the right index finger in the state of resting sitting position. The PPG pulse waves of 12 hours were measured using PowerLab 4/26 (ADinstruments, Sydney, Australia) as the measuring instrument. The systolic BPs of twice every 5 minutes were measured with a cuff using HEM-1020 (Omron Healthcare Co. Ltd., Kyoto, Japan) and the average of the systolic BP was taken as the estimation. By the above operation, data sets of $144 \mathrm{BP}$ values and pulse wave data are obtained.

The pulse wave data of 30 seconds $\times 6$ sections are equally divided from 3 minutes before each BP measurement time point as shown in Figure 7. The multiple regression analysis is performed using proposed algorithm to select parameters and designate the optimum section out of 6 sections.

Figure 8 shows the experiment procedure as follows: 


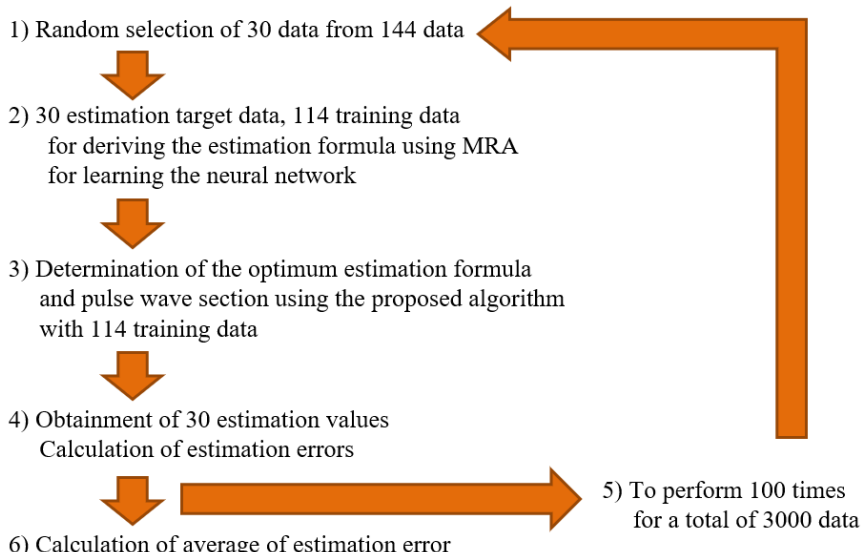

Figure 8. Experiment procedure.

1) 30 data are randomly selected from 144 data. These 30 pieces are selected again each time the procedure of loop 1) to 4).

2) The selected 30 pieces data are the estimation target data. And the remaining 114 pieces data are used as the training data for deriving the estimation formula using multiple regression analysis and for learning the neural network.

3) In 6 sections of the 114 training data, the proposed algorithm using multiple regression analysis is performed to determine the optimum estimation formula with the largest coefficient of determination. Moreover, section of the pulse wave from which the estimated expression was derived is defined as optimum pulse wave section.

4) The BP estimation are performed by using 114 training data and total of 4 type methods of the multiple regression analysis and the neural network estimations. Thirty estimation values are obtained for each. In each method, 30 estimation errors are calculated from estimated values and correct values.

5) 100 times are performed above 1) to 4).

6) The average of estimation error is calculated for each method from a total of 3,000 data.

\subsection{Experimental Result}

The estimation accuracy of the BP estimation using the multiple regression analysis and that using the neural network are compared. The MRA_A is a conventional method using all 16 parameters [15] and the MRA_B is using the proposed algorithm. Then, the NN Esti_A model estimates with the neural network learned easily using 16 parameters of all training data, however, does not use the proposed algorithm. Furthermore, the NN Esti_B model is using designation of the optimum section. Table 2 shows conditions of learning type using the proposed
Table 2. Conditions of learning type

\begin{tabular}{lcc}
\hline & $\begin{array}{c}\text { Selection of } \\
\text { parameters }\end{array}$ & $\begin{array}{c}\text { Designation of } \\
\text { sections }\end{array}$ \\
\hline MRA_A & $\times$ & $\times$ \\
MRA_B & $\circ$ & $\circ$ \\
NN Esti_A & $\times$ & $\times$ \\
NN Esti_B & $\times$ & $\circ$ \\
\hline
\end{tabular}

algorithm.

Comparing the average of estimation error in each method is shown in Figure 9. Here, the $n$ represents the number of effective estimations among 100 estimation formulas. In the estimation method using multiple regression analysis, the average value of estimation error of the MRA_B using the proposed algorithm was smaller than that of the MRA_A. Moreover, the number of times $n$ where effective estimation formula could be derived was large. Estimation error of the MRA_B was reduced by approximately $16.3 \%$ compared with the conventional method. Comparing the MRA_A with the NN Esti_A which did not perform parameter selection and optimum section designation, the estimation error of the NN Esti_A was approximately $21.6 \%$ smaller, and the effective number of times $n$ was larger than that of the MRA_A. Furthermore, comparing the MRA_A with the NN Esti_B which only performed optimum section designation, the estimation error of the NN Esti_B was approximately $20.0 \%$ smaller, and the effective number of times $n$ was larger than that of the MRA_A. Comparing the MRA_B with the NN Esti_A, the estimation error decreases without using the proposed algorithm because of estimating with the neural network's learning ability. Although the effective number of times $n$ of the NN Esti_A was lower than that of the MRA_B, the $n$ of the NN Esti_B using the proposed algorithm was higher than any of them. Furthermore, it is considered that the number $n$ becomes high when the amount of training data in the neural network learning increases.

Experimental results have shown that estimation accuracy is improved. In addition, the estimation error can be reduced when applying the algorithm considering the characteristics of the individual rather than the general method as shown in Figure 9, although experiments and analyzes using data of one subject were performed in this study. In the future, we will increase the number of subjects and verify the proposed algorithm. 


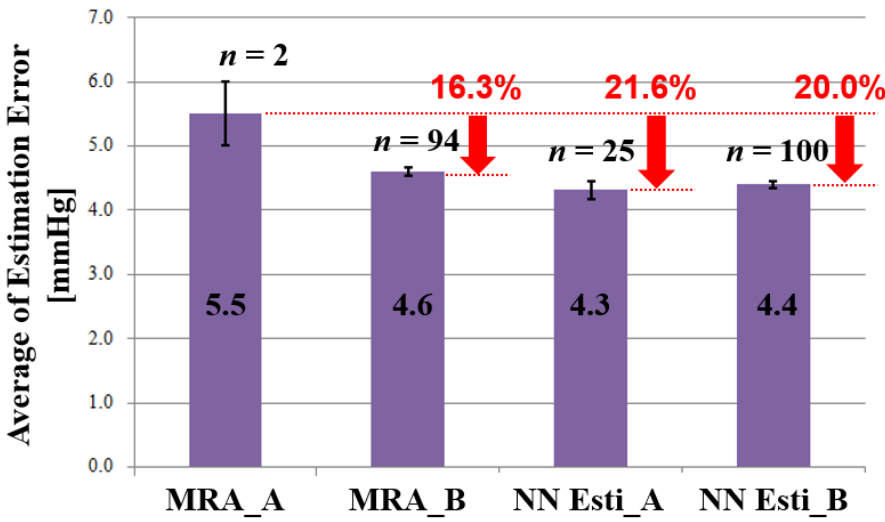

Figure 9. Comparing the average of estimation error in each method.

\section{Conclusion}

The goal is to establish a method to estimate the BP value using the PPG signals. In this study, an improvement algorithm of the BP estimation method was proposed and verified using multiple regression analysis. Furthermore, BP estimation was investigated using the neural network with/without designation of sections.

It was possible to raise the number $n$ which effective estimation formulas were automatically derived using the proposed algorithm. Moreover, the proposed system reduced the estimation error of the systolic BP value by multiple regression analysis and improved the estimation accuracy. In addition, the estimation error using the neural network was lower than that using conventional multiple regression analysis and improves the estimation accuracy because BP estimation was performed based on machine learning using the neural network. It is found the possibility of estimation BP using the neural network.

\section{Conflict of Interest}

No potential conflict of interest relevant to this article was reported.

\section{Acknowledgments}

This paper is a revised and expanded version of a paper entitled "Estimation System of Blood Pressure Variation Using Multiple Regression Analysis and Neural Network" presented at the 7th International Conference on Next Generation Computer and Information Technology, Hokkaido, Japan, August 23-25, 2018.

This work was partly supported by JSPS KAKENHI (Granted Number JP17H01729, JP15H03699, 18K11459, 15K00330, and $18 \mathrm{H} 03303)$. Beside this work was also partly supported by the Center of Innovation Program from the Japan Science and Technology Agency (JST) and JST CREST (Grant Number JPMJCR1402).

\section{References}

[1] T. Shima, K. Seki, A. Umemura, R. Ogawa, R. Horimoto, H. Oya, R. Sendo, M. Mizuno, and T. Okanoue, "Influence of lifestyle-related diseases and age on the development and progression of non-alcoholic fatty liver disease," Hepatology Research, vol. 45, no. 5, pp. 548-559, 2015. https://doi.org/10.1111/hepr.12384

[2] J. Dieffenderfer, H. Goodell, S. Mills, M. McKnight, S. Yao, F. Lin, et al., "Low-power wearable systems for continuous monitoring of environment and health for chronic respiratory disease," IEEE Journal of Biomedical and Health Informatics, vol. 20, no. 5, pp. 1251-1264, 2016. https://doi.org/10.1109/JBHI.2016.2573286

[3] N. C. Gassen, G. P. Chrousos, E. B. Binder, and A. S. Zannas, "Life stress, glucocorticoid signaling, and the aging epigenome: implications for aging-related diseases," Neuroscience and Biobehavioral Reviews, vol. 74, pp. 356-365, 2017. https://doi.org/10.1016/j.neubiorev.2016. 06.003

[4] T. Niccoli and L. Partridge, "Ageing as a risk factor for disease," Current Biology, vol. 22, no. 17, pp. R741-R752, 2012. https://doi.org/10.1016/j.cub.2012.07.024

[5] T. Sekine, R. Sugano, T. Tashiro, J. Sato, Y. Takeda, H. Matsui, D. Kumaki, F. D. Dos Santos, A. Miyabo, and S. Tokito, "Fully printed wearable vital sensor for human pulse rate monitoring using ferroelectric polymer," Scientific Reports, vol. 8, article no. 4442, 2018. https://doi.org/10.1038/s41598-018-22746-3

[6] 1. chen and v. b. surya prasath, "measuring bone density connectivity using dual energy X-ray absorptiometry images," International Journal of Fuzzy Logic and Intelligent Systems, vol. 17, no. 4, pp. 235-244, 2017. https://doi.org/10.5391/IJFIS.2017.17.4.235

[7] E. Urtnasan, J. U. Park, S. Lee, and K. J. Lee, “optimal classifier for detection of obstructive sleep apnea using a heartbeat signal," International Journal of Fuzzy Logic and Intelligent Systems, vol. 17, no. 2, pp. 76-81, 2017. https://doi.org/10.5391/IJFIS.2017.17.2.76 
[8] W. Gao, S. Emaminejad, H. Y. Y. Nyein, S. Challa, K. Chen, A. Peck, et al., "Fully integrated wearable sensor arrays for multiplexed in situ perspiration analysis," Nature, vol. 529, no. 7587, pp. 509-514, 2016. https://doi.org/10.1038/nature16521

[9] T, Yokota, P. Zalar, M. Kaltenbrunner, H. Jinno, N. Matsuhisa, H. Kitanosako, Y. Tachibana, W. Yukita, M. Koizumi, and T. Someya, "Ultraflexible organic photonic skin," Science Advances, vol. 2, no. 4, article no. e1501856, 2016. https://doi.org/10.1126/sciadv.1501856

[10] R. E. Ogunsakin, R.B. Ogunrinde, O. Omotoso, and O. B. Adewale, "On regression analysis of the relationship between age and blood cholesterol on blood pressure," International Journal of Scientific \& Technology Research, vol. 1, no. 9, pp. 92-94, 2012.

[11] J. D. Bundy, C. Li, P. Stuchlik, X. Bu, T. N. Kelly, K. T. Mills, H. He, J. Chen, P. K. Whelton, and J. He, "Systolic blood pressure reduction and risk of cardiovascular disease and mortality: a systematic review and network meta-analysis," JAMA Cardiology, vol. 2, no. 7, pp. 775781, 2017. https://doi.org/10.1001/jamacardio.2017.1421

[12] E. Rapsomaniki, A. Timmis, J. George, M. PujadesRodriguez, A. D Shah, S. Denaxas, et al., "Blood pressure and incidence of twelve cardiovascular diseases: lifetime risks, healthy life-years lost, and age-specific associations in 125 million people," The Lancet, vol. 383, no. 9932, pp. 1899-1911, 2014. https://doi.org/10.1016/S0140-6736(14) 60685-1

[13] K. Atomi, H. Kawanaka, M. S. Bhuiyan, and K. Oguri, "Cuffless blood pressure estimation based on data-oriented continuous health monitoring system," Computational and Mathematical Methods in Medicine, vol. 2017, article ID. 1803485, 2017. https://doi.org/10.1155/2017/1803485

[14] M. Kachuee, M. M. Kiani, H. Mohammadzade, and M Shabany, "Cuffless blood pressure estimation algorithms for continuous health-care monitoring," IEEE Transactions on Biomedical Engineering, vol. 64, no. 4, pp. 859869, 2016. https://doi.org/10.1109/TBME.2016.2580904

[15] M. Yokoyama, T. Negishi, M. Mizunuma, K. Otani, H. Hanaki, and K. Nishimura, "Multiple regression analysis and learning system for estimation of blood pressure variation using photo-plethysmograph signals," International
Journal of Computers and Their Applications, vol. 23, no. 1, pp. 12-18, 2016.

[16] J. S. Kim, "Vessel target prediction method and dead reckoning position based on SVR seaway model," International Journal of Fuzzy Logic and Intelligent Systems, vol. 17, no. 4, pp. 279-288, 2017. https://doi.org/10.5391/ IJFIS.2017.17.4.279

[17] S. I. Cho, T. Negishi, M. Yasuda, and M. Yokoyama, "Estimation system of blood pressure variation with photoplethysmograph signals using neural network," in Proceedings of 30th International Conference on Computer Applications in Industry and Engineering (CAINE), San Diego, CA, 2017, pp. 155-160.

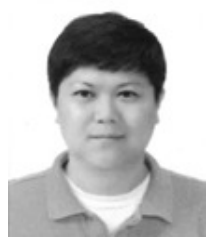

Seung-Il Cho received his B.S. and M.S. degrees in marine electronics and communication engineering from Mokpo National Maritime University, Korea in 2006 and 2008, respectively. He was an associate research engineer in the R\&D division of Tomato LSI from 2008 to 2010 He received his Ph.D. degree in bio-systems engineering, Graduate School of Science and Engineering, Yamagata University, Japan in 2013. He is currently an Assistant Professor in the Innovation Center for Organic Electronics, Yamagata University. His research interests include adiabatic dynamic CMOS logic (ADCL), digital/analog integrated circuit design, organic thin film transistor (OTFT) circuit design, organic light emitting diode (OLED) flexible displays, ubiquitous healthcare system, and Information and Communications Technologies (ICT). He is a member of The Korea Institute of Electronic Communication Sciences.

E-mail: cho_si@yz.yamagata-u.ac.jp

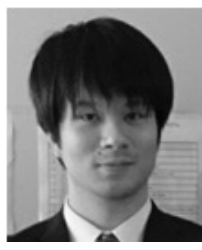

Takumi Negishi received his B.E. degree in bio-system engineering from Yamagata University in 2014. He received his M.E. degree in bio-system engineering at Graduate School of Science and Engineering, Yamagata University in 2016. Since 2016, he joined SCSK corporation. His research interests include ubiquitous healthcare system, vital signal sensing and analysis.

E-mail: tes77093@st.yamagata-u.ac.jp 


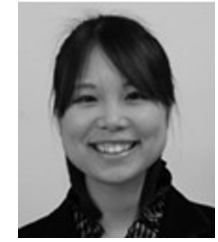

Minami Tsuchiya received her B.E. degree in bio-systems engineering from Yamagata University in 2016. She is currently a student for $\mathrm{Ph} . \mathrm{D}$. degree in bio engineering at Graduate School of Science and Engineering, Yamagata University. She also belongs to Innovative Flex Course for Frontier Organic Material Systems (iFront), 5 years integrated doctoral course of Yamagata University. Her research interests include vital sensing and analysis, ubiquitous health care system, preventive medicine, self-medication, and Information and Communications Technologies (ICT).

E-mail: tsc84435@st.yamagata-u.ac.jp

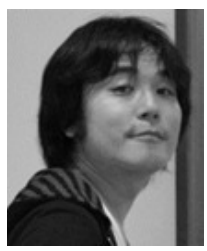

Muneki Yasuda received the doctoral degree (information science) from Tohoku University in 2007. He worked at Tohoku University from 2007 to 2013 as an Assistant Professor. He has worked in Yamagata University as an Associate Professor since 2013. His research interests are statistical machine learning, data mining, artificial intelligence (AI), signal processing, and statistical mechanics.

E-mail: muneki@yz.yamagata-u.ac.jp

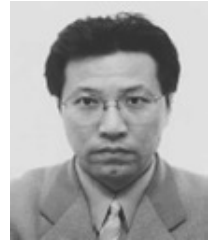

Michio Yokoyama received his B.E. degree in electrical engineering from Yamagata University in 1989, and his M.E. degree in electrical and communication engineering and Ph.D. degree in electronic engineering from Tohoku University in 1991 and 1994, respectively. From 1994 to 2001, he joined the Research Institute of Electrical Communication (RIEC), Tohoku University, Sendai, Japan, where he was engaged in research on the design and development of RF-CMOS devices, such as power amplifier modules for digital cellular phone systems. Since 2001, he has joined Yamagata University, Yonezawa, Japan, and was engaged in research on ubiquitous healthcare system, Information and Communications Technologies (ICT), Internet of Things (IoT). He is a member of The Japan Institute of Electronics Packaging, The Japan Society of Applied Physics, The Institute of Electrical Engineers of Japan and The Institute of Electronics, Information and Communication Engineers.

E-mail: yoko@yz.yamagata-u.ac.jp 